\section{Case Reports in Oncology}

\title{
First-Line Treatment with Carboplatin plus nab-Paclitaxel and Maintenance Monotherapy with nab-Paclitaxel for a Thymic Carcinoma: A Case Report
}

\author{
Kunihiko Funaishi ${ }^{\mathrm{a}}$ Masahiro Yamasaki ${ }^{\mathrm{a}}$ Naomi Saito ${ }^{\mathrm{b}}$ Wakako Daido \\ Sayaka Ishiyama ${ }^{a}$ Naoko Deguchi ${ }^{a}$ Masaya Taniwaki ${ }^{a}$ \\ Nobuyuki Ohashi ${ }^{\mathrm{a}, \mathrm{c}}$ \\ ${ }^{a}$ Department of Respiratory Disease, Hiroshima Red Cross Hospital \& Atomic Bomb \\ Survivors Hospital, Hiroshima, Japan; ${ }^{b}$ Department of Respiratory Medicine, \\ Mazda Hospital, Hiroshima, Japan; 'Ohashi Clinic, Hiroshima, Japan
}

\section{Keywords}

Carboplatin · nab-Paclitaxel $\cdot$ Maintenance $\cdot$ First-line treatment $\cdot$ Thymic carcinoma

\section{Abstract}

Thymic carcinomas are rare malignant tumors, located in the anterior mediastinum. For the treatment of these carcinomas, several chemotherapy regimens have been suggested, including carboplatin plus paclitaxel. However, because of the rarity of these tumors, the standard chemotherapy regimen has not yet been established. Here, we report a case of thymic carcinoma that responded to first-line carboplatin plus nanoparticle albumin-bound paclitaxel (nab-paclitaxel) therapy with continuation maintenance $n a b$-paclitaxel monotherapy. A 78-year-old male presented to a hospital with the chief complaint of dyspnea. Cardiomegaly was detected on chest X-ray scans, and marked pericardial effusion was observed by echocardiography. Chest computed tomography scans revealed the presence of a mediastinal mass, pericardial thickening, and pericardial effusion. The serum levels of the tumor marker CYFRA 21-1 (cytokeratin-19 fragment) were elevated. Eventually, he was diagnosed 


\section{Case Reports in Oncology}

with squamous cell carcinoma of the thymus, which was staged as CT4N3M0 or stage IV (according to the tumor-node-metastasis classification). Chemotherapy with carboplatin on day 1 and nab-paclitaxel on days 1,8 , and 15, every 4 weeks was initiated. After the administration of 4 cycles of this regimen, the tumor diameter appeared reduced, and the serum CYFRA 21-1 levels were normalized. After a 1-month interval, the serum CYFRA 21-1 levels increased again; therefore, maintenance nab-paclitaxel monotherapy was initiated. At the end of the treatment, the patient experienced a progression-free survival of 10.3 months. Carboplatin plus nab-paclitaxel may be an appropriate alternative first-line treatment for thymic carcinomas. Additionally, maintenance nab-paclitaxel monotherapy may prolong the progressionfree survivals of patients with thymic carcinomas.

(C) 2017 The Author(s)

Published by S. Karger AG, Basel

\section{Introduction}

Thymic carcinomas are rare malignant tumors located in the anterior mediastinum. These tumors arise from the thymic epithelium and have been reported to account for $12-36 \%$ of all thymic epithelial tumors [1-3]. Complete surgical resection is the preferred method of treatment for thymic carcinomas with no distant metastases. In cases with unresectable tumors or distant metastases, systemic chemotherapy is administered. Several chemotherapy regimens have been suggested, such as the ADOC (cisplatin, doxorubicin, vincristine, and cyclophosphamide) [4], CODE (cisplatin, vincristine, doxorubicin, and etoposide) [5], and carboplatin plus paclitaxel [6] regimens. However, because of the rarity of these tumors, the standard regimen of chemotherapy has not yet been established.

Here, we report a case of thymic carcinoma that responded to treatment with a carboplatin plus nanoparticle albumin-bound paclitaxel ( $n a b$-paclitaxel) regimen; moreover, continuation maintenance monotherapy with $n a b$-paclitaxel may have enabled a prolonged progression-free survival in this patient.

\section{Case Presentation}

A 78-year-old male presented to a hospital with the chief complaints of dyspnea and ache in his left shoulder. Cardiomegaly was detected on chest X-ray scans, and the presence of pericardial effusion and moderate aortic regurgitation was observed by echocardiography. He was treated with diuretic agents, and his symptoms improved. Three months later, he presented to the hospital again with worsening dyspnea. Worsening cardiomegaly was detected on chest X-ray scans (Fig. 1a), and marked pericardial effusion was observed on echocardiography. Pericardiocentesis was performed, and 1,500 mL of hemorrhagic pericardial effusion fluid was drained. Chest examination by computed tomography revealed the presence of a mediastinal mass, pericardial thickening, and pericardial effusion (Fig. 1b, c), and the patient was transferred to our hospital for treatment. He showed marked emaciation (he was $164.6 \mathrm{~cm}$ tall and weighed $43.6 \mathrm{~kg}$ ) and a low blood pressure (81/47 $\mathrm{mm} \mathrm{Hg}$ ). He was assessed to have an Eastern Cooperative Oncology Group (ECOG) performance status (PS) of 2. His laboratory findings from a complete blood cell count analysis were almost normal; his hepatic and renal functions were normal; his albumin levels were lower than normal $(2.7 \mathrm{mg} / \mathrm{dL})$, and his C-reactive protein levels were high $(10.00 \mathrm{mg} / \mathrm{dL})$. The levels of the soluble serum tumor marker CYFRA 21-1 (cytokeratin-19 fragment) were increased $(36.0 \mathrm{ng} / \mathrm{mL})$. After further examination, he was diagnosed with squamous cell carcinoma of 


\section{Case Reports in Oncology}

the thymus. According to the tumor-node-metastasis staging system, the carcinoma stage was cT4N3M0 or stage IV.

Carboplatin was administered on day 1 at a dose of a targeted area under the concentration-time curve of 5, and $n a b$-paclitaxel at a dose of $70 \mathrm{mg} / \mathrm{m}^{2}$ on days 1,8 , and 15 for every 4 weeks. Four cycles of this regimen were administered. During 3 of 4 cycles, grade 3 or 4 neutropenia occurred, and 3 doses of nab-paclitaxel on day 15 or 8 could not be administered. The patient did not experience any other severe adverse events. After the administration of 2 cycles of this regimen, chest computed tomography scans revealed a reduction of the tumor diameter (Fig. 2a); administration of 2 additional cycles led to further reductions (Fig. 2b). The ECOG PS of the patient improved to 0 (zero). The serum CYFRA 21-1 levels decreased to $2.3 \mathrm{ng} / \mathrm{mL}$. After the interval of 1 month, these levels increased again to 4.3 $\mathrm{ng} / \mathrm{mL}$. Resultantly, maintenance nab-paclitaxel monotherapy at a dosage of $70 \mathrm{mg} / \mathrm{m}^{2}$ on days 1 and 8, for every 3 weeks was initiated. A total of 8 cycles of maintenance nabpaclitaxel monotherapy were administered in 4.8 months, after which the tumor showed regrowth. The patient experienced a total of 10.3 months of progression-free survival from the start of chemotherapy. After the regrowth of the tumor, the patient has received secondline treatment. Till date, the patient has been alive for a total of 22.0 months from the start of first-line chemotherapy.

\section{Discussion}

In the present case report, we noted 2 important clinical observations: (1) the carboplatin plus nab-paclitaxel regimen may be effective as a first-line treatment for thymic carcinomas, and (2) maintenance nab-paclitaxel monotherapy might prolong the progression-free survival in patients with thymic carcinomas. These observations are further discussed in-depth.

Firstly, our study suggests that carboplatin plus nab-paclitaxel may be effective as firstline treatment for thymic carcinomas. To the best of our knowledge, this is the first report describing the efficacy of such treatment. Till date, carboplatin plus nab-paclitaxel therapy has proved to be efficacious in 3 cases of thymic carcinomas that have been reported in English journals; however, all of these cases had undergone previous treatments [7-9]. Some retrospective [10] and prospective [6] studies have reported that carboplatin plus paclitaxel has been effective for the treatment of thymic carcinomas. However, $n a b$-paclitaxel is a nanoparticle albumin-bound formulation of paclitaxel. This new generation paclitaxel has been more effective in the treatment of non-small cell lung cancers (NSCLCs); moreover, it is less toxic, in terms of the incidence of peripheral neuropathy, neutropenia, arthralgia, and myalgia [11]. For NSCLCs, the doses of carboplatin and nab-paclitaxel are as follows: carboplatin at a dose of an area under the concentration-time curve of 6 on day 1 and nab-paclitaxel at a dose of $100 \mathrm{mg} / \mathrm{m}^{2}$ on days 1,8 , and 15 for every 3 weeks [11]. In the present case, the doses of carboplatin and nab-paclitaxel had to be reduced because the patient was elderly and had poor PS. Nevertheless, the treatment was effective. Thus, we conclude that carboplatin plus nab-paclitaxel therapy may be a good alternative first-line treatment for thymic carcinomas.

Secondly, our study also suggests that maintenance nab-paclitaxel monotherapy might prolong the progression-free survivals of patients with thymic carcinomas. In NSCLCs, the administration of maintenance paclitaxel monotherapy after carboplatin plus paclitaxel treatment prolonged the progression-free and overall survivals in treated patients [12]. Resultantly, the use of maintenance paclitaxel monotherapy was adopted at a phase III trial 


\section{Case Reports in Oncology}

comparing carboplatin plus weekly paclitaxel with carboplatin plus standard paclitaxel for the treatment of NSCLCs [13]. In the present case of thymic carcinoma, nab-paclitaxel monotherapy maintained a stable condition for the disease for 5.5 months. Thus, we conclude that maintenance $n a b$-paclitaxel monotherapy after carboplatin plus $n a b$-paclitaxel treatment might prolong the progression-free survivals of patients with thymic carcinomas.

For the treatment of thymic carcinomas, the continuation of carboplatin plus nabpaclitaxel could be better than the administration of maintenance nab-paclitaxel monotherapy. At the phase III study of NSCLCs, the cycles of carboplatin plus nab-paclitaxel could be continued till the time of disease progression [11]. However, it has been demonstrated that the patients with advanced NSCLCs did not show improved overall survivals during the administration of 6 cycles of first-line platinum-based chemotherapy, compared with the administration of 3 or 4 cycles of such regimens [14]. Moreover, the accumulation of carboplatin may cause hypersensitivity reactions in the patients [15]. For cases in which the continued administration of carboplatin is difficult because of the occurrence of adverse events, continuation maintenance $n a b$-paclitaxel monotherapy might prove to be an appropriate alternative treatment.

It is uncertain whether carboplatin plus $n a b$-paclitaxel therapy is effective for all histopathological subtypes of thymic carcinomas. These histopathological subtypes are as follows: squamous cell carcinomas; neuroendocrine carcinomas, including small-cell and largecell neuroendocrine carcinomas, and carcinoid tumors; mucoepidermoid carcinomas; and others [1]. The histopathological subtypes of the 3 cases previously reported were as follows: squamous cell carcinoma [7], large-cell neuroendocrine carcinoma [8], and lymphoepithelioma-like carcinoma [9]; all of them were reported to be effectively treated with carboplatin plus $n a b$-paclitaxel. It has also been reported that carboplatin plus $n a b$-paclitaxel was more effective for the treatment of squamous cell carcinoma than that of non-squamous cell carcinomas in NSCLCs [11]. A prospective study is needed to evaluate the efficacy of carboplatin plus nab-paclitaxel treatment for thymic carcinomas of all known histological subtypes.

\section{Conclusions}

We report a case of thymic carcinoma treated with first-line carboplatin plus nabpaclitaxel with maintenance $n a b$-paclitaxel monotherapy. We conclude that carboplatin plus $n a b$-paclitaxel may be a good alternative first-line treatment for thymic carcinomas. Additionally, maintenance nab-paclitaxel monotherapy might prolong the progression-free survivals of patients with thymic carcinomas.

\section{Acknowledgement}

We would like to thank Editage (www.editage.jp) for English language editing.

\section{Statement of Ethics}

The authors have no ethical conflicts to disclose.

Written consent was obtained from the patient for the publication of this case report. 


\section{Disclosure Statement}

The authors declare that they have no conflicts of interest to disclose.

\section{References}

1 Okuma Y, Hosomi Y, Watanabe K, Yamada Y, Horio H, Maeda Y, Okamura T, Hishima T:

Clinicopathological analysis of thymic malignancies with a consistent retrospective database in a single institution: from Tokyo Metropolitan Cancer Center. BMC Cancer 2014;14:349.

2 Nakajima J, Okumura M, Yano M, Date H, Onuki T, Haniuda M: Myasthenia gravis with thymic epithelial tumour: a retrospective analysis of a Japanese database. Eur J Cardiothorac Surg 2016;49:1510-1515.

-3 Kondo K, Monden Y: Therapy for thymic epithelial tumors: a clinical study of 1,320 patients from Japan. Ann Thorac Surg 2003;76:878-885.

-4 Koizumi T, Takabayashi Y, Yamagishi S, Tsushima K, Takamizawa A, Tsukadaira A, Yamamoto H, Yamazaki Y, Yamaguchi S, Fujimoto K, Kubo K, Hirose Y, Hirayama J, Saegusa H: Chemotherapy for advanced thymic carcinoma: clinical response to cisplatin, doxorubicin, vincristine, and cyclophosphamide (ADOC chemotherapy). Am J Clin Oncol 2002;25:266-268.

-5 Yoh K, Goto K, Ishii G, Niho S, Ohmatsu H, Kubota K, Kakinuma R, Nagai K, Suga M, Nishiwaki Y: Weekly chemotherapy with cisplatin, vincristine, doxorubicin, and etoposide is an effective treatment for advanced thymic carcinoma. Cancer 2003;98:926-931.

-6 Hirai F, Yamanaka T, Taguchi K, Daga H, Ono A, Tanaka K, Kogure Y, Shimizu J, Kimura T, Fukuoka J, Iwamoto Y, Sasaki H, Takeda K, Seto T, Ichinose Y, Nakagawa K, Nakanishi Y; West Japan Oncology Group: A multicenter phase II study of carboplatin and paclitaxel for advanced thymic carcinoma: WJOG4207L. Ann Oncol 2015;26:363-368.

7 Makimoto G, Fujiwara K, Watanabe H, Kameyama N, Matsushita M, Rai K, Sato K, Yonei T, Sato T, Shibayama T: nab-Paclitaxel in combination with carboplatin for a previously treated thymic carcinoma. Case Rep Oncol 2014;7:14-17.

-8 Igawa S, Yanagisawa N, Niwa H, Ishihara M, Hiyoshi Y, Otani S, Katono K, Sasaki J, Satoh Y, Masuda N: Successful chemotherapy with carboplatin and nab-paclitaxel for thymic large cell neuroendocrine carcinoma: a case report. Oncol Lett 2015;10:3519-3522.

-9 Shima H, Ozasa H, Tsuji T, Ajimizu H, Nomizo T, Yagi Y, Sakamori Y, Nagai H, Minamiguchi S, Kim YH, Mishima M: Response to chemotherapy with carboplatin plus albumin-bound paclitaxel in a patient with lymphoepithelioma-like thymic carcinoma: a case report. Mol Clin Oncol 2016;4:715-718.

10 Igawa S, Murakami H, Takahashi T, Nakamura Y, Tsuya A, Naito T, Kaira K, Ono A, Shukuya T, Tamiya A, Endo M, Yamamoto N: Efficacy of chemotherapy with carboplatin and paclitaxel for unresectable thymic carcinoma. Lung Cancer 2010;67:194-197.

-11 Socinski MA, Bondarenko I, Karaseva NA, Makhson AM, Vynnychenko I, Okamoto I, Hon JK, Hirsh V, Bhar P, Zhang H, Iglesias JL, Renschler MF: Weekly nab-paclitaxel in combination with carboplatin versus solvent-based paclitaxel plus carboplatin as first-line therapy in patients with advanced nonsmall-cell lung cancer: final results of a phase III trial. J Clin Oncol 2012;30:2055-2062.

12 Belani CP, Barstis J, Perry MC, La Rocca RV, Nattam SR, Rinaldi D, Clark R, Mills GM: Multicenter, randomized trial for stage IIIB or IV non-small-cell lung cancer using weekly paclitaxel and carboplatin followed by maintenance weekly paclitaxel or observation. J Clin Oncol 2003;21:2933-2939.

13 Belani CP, Ramalingam S, Perry MC, LaRocca RV, Rinaldi D, Gable PS, Tester WJ: Randomized, phase III study of weekly paclitaxel in combination with carboplatin versus standard every-3-weeks administration of carboplatin and paclitaxel for patients with previously untreated advanced nonsmall-cell lung cancer. J Clin Oncol 2008;26:468-473.

14 Rossi A, Chiodini P, Sun JM, O’Brien ME, von Plessen C, Barata F, Park K, Popat S, Bergman B, Parente B, Gallo C, Gridelli C, Perrone F, Di Maio M: Six versus fewer planned cycles of first-line platinum-based chemotherapy for non-small-cell lung cancer: a systematic review and meta-analysis of individual patient data. Lancet Oncol 2014;15:1254-1262.

15 Sliesoraitis S, Chikhale PJ: Carboplatin hypersensitivity. Int J Gynecol Cancer 2005;15:13-18. 


\section{Case Reports in Oncology}

www.karger.com/cro

Funaishi et al: First-Line Treatment with Carboplatin plus nab-Paclitaxel and Maintenance Monotherapy with nab-Paclitaxel for a Thymic Carcinoma: A Case Report
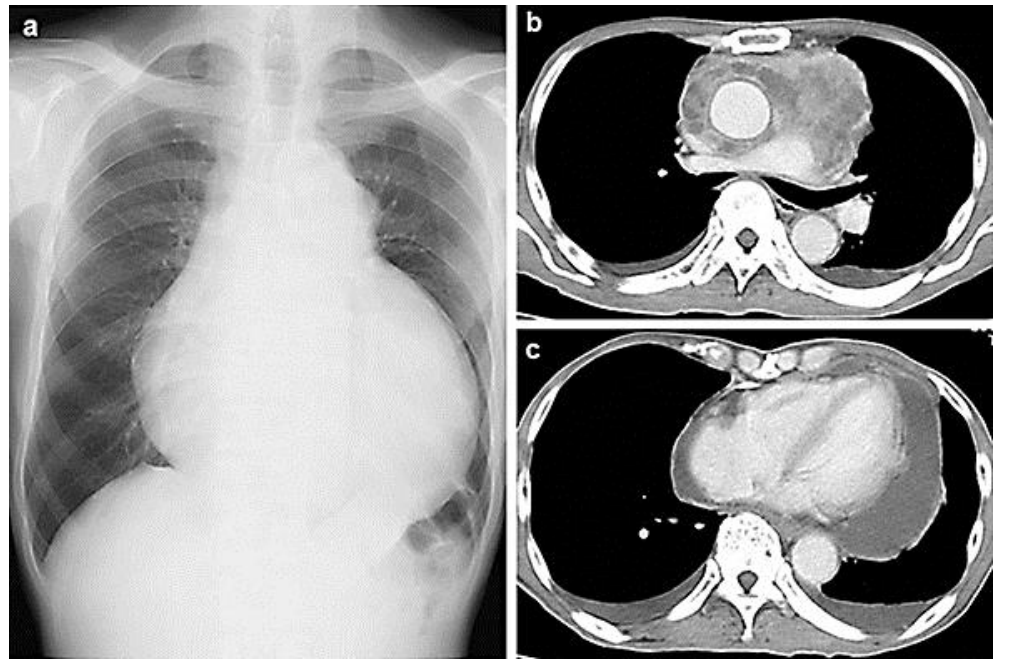

Fig. 1. Initial chest X-ray and chest computed tomography scans. a Significant cardiomegaly is present. b An anterior mediastinal mass can be observed on chest computed tomography scans after pericardiocentesis. c Pericardial effusion and pericardial thickening suggest the presence of carcinomatous pericarditis.
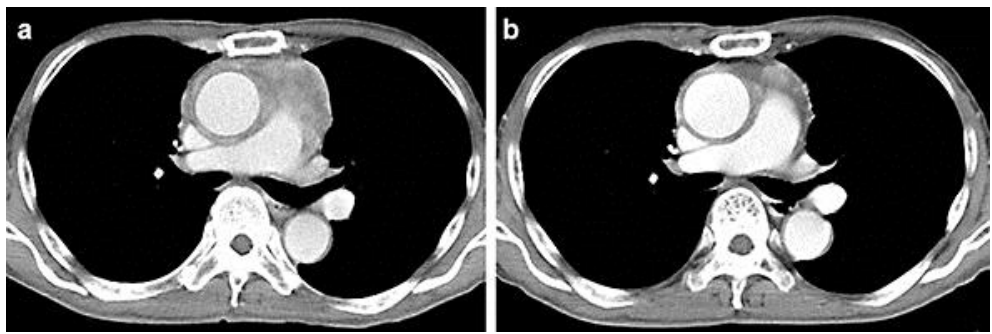

Fig. 2. The findings of chest computed tomography scans after the administration of 2 and 4 cycles of carboplatin plus nab-paclitaxel therapy. a Chest computed tomography scan obtained after the administration of 2 cycles of the regimen. The tumor appears reduced in diameter. $\mathbf{b}$ Chest computed tomography scan taken after the administration of 4 cycles of the regimen. The tumor appears markedly reduced in diameter. 